\title{
Intertrochanteric Fractures: Ten Commandments for How to Get Good Results with Proximal Femoral Nailing
}

\author{
B. Shivashankar ${ }^{1} \cdot$ Sanjay Keshkar ${ }^{2}$
}

Published online: 16 March 2021

C Indian Orthopaedics Association 2021

Intertrochanteric (IT) fractures in the elderly is a challenging surgery. The bones are osteoporotic with implants having less hold as well as the co morbidities associated in these elderly leads to almost one-third mortality by the end of one year, which is a major global economic burden. The hip fractures accounted for over 250,000 fractures in US itself every year [1]. The worldwide problem at present stands at over 2 million fractures and it is bound to triple by the next 50 years due to the elderly surviving more with better healthcare facilities. The treatment option for osteosynthesis includes open/closed reduction and fixation either with surface implants such as dynamic hip screw (DHS) or intramedullary (IM) implants such as proximal femoral nail (PFN) [2, 3].

Approximate period between 1999 and 2010, most of the studies [3, 4] and the Cochrane reviews were advising DHS or the sliding Hip screw for Stable IT fractures whereas for unstable fractures, the IM implants were advocated. Now, most of the studies $[5,6]$ including the recent Cochrane data advocates the use of IM hip implants both for stable and unstable fracture. The author also advocates the same.

As the author referrers IM implants, here are few tips and tricks to get a better outcome with IM implants (PFN).

1. Good anterior reduction and temporary fixation by two K-Wires Sometimes we may not get an acceptable reduction on the orthopaedic table easily and in such cases, the reduction can be assisted either by manipulating the fragment by closed techniques or by per-

B. Shivashankar

drbshivashankar@gmail.com

Sanjay Keshkar

s_keshkar@yahoo.co.in

1 Iyer Orthopaedic Centre, 103, Railway Lines, Solapur, Maharashtra 413001, India

2 ESIC Medical College and Hospital, Joka, Kolkata 700104, India cutaneous mini-open techniques such as using a bone lever, Bone spatula, bone hook, and collinear clamps. To add on it, the author's preference is almost always intrafocal manipulation with Steinmann pin by passing at the fracture site and lifting the depressed fragment (either the neck or the shaft). Once anterior cortex is well aligned, percutaneouly it is fixed with two Kirschner's wires which are very anteriorly placed. Once the fracture is fixed temporarily with $\mathrm{K}$ wires, the hip can be adducted without fracture going into varus, making nailing an easy procedure (Fig. 1a).

2. Good medial reduction (positive cortical reduction) Valgoid reduction of at least 5 degrees more in valgus compared to the normal side is also very important. In valgoid reduction make sure that neck beak is outside the distal fragment (positive cortical reduction) and should not enter into the shaft (negative cortical support) [7]. One should aim at neutral with valgus or Positive cortical reduction. This can be done by intrafocal Steinmann pin pushing the beak out medially and reducing the foot traction minimally so that beak will overlap medially on the beak of the shaft and it can never slip back inside the distal canal due to this few mm overlap (Fig. 1b). This is more important if the lesser trochanter is avulsed. Due to good valgus, even though there is void medially, bone forms in due course without any hindrance. If there is a large medial fragment of lesser trochanter extending into the subtrochanteric area, the author prefers to fix them with encirclage wiring. In just avulsion of lesser trochanter, even if you do not open or reduce it into its bed, a good valgus compensates for the medial void very well.

3. The entry point It has to be done in line with the medullary canal, which is almost always either the piriformis fossa (Which is a misnomer as this is much more posterior in the lateral view and not in the central part, this area is medial to the tip of trochanter at its base) or medial to the tip of the trochanter (Fig. 1c). 


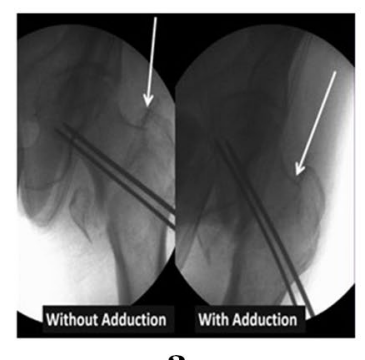

a

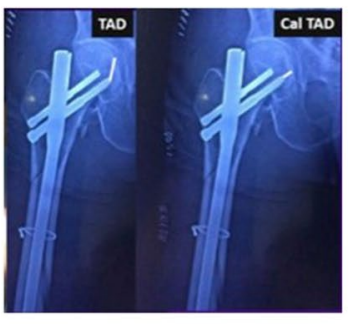

e

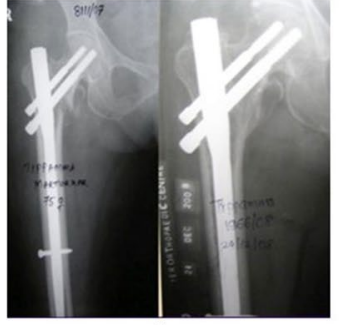

b

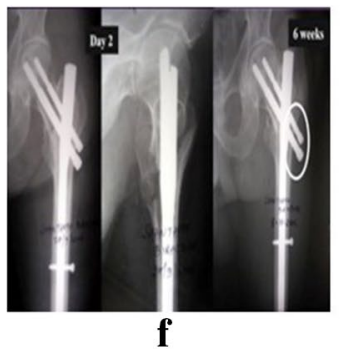

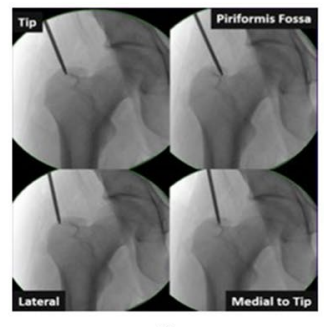

c

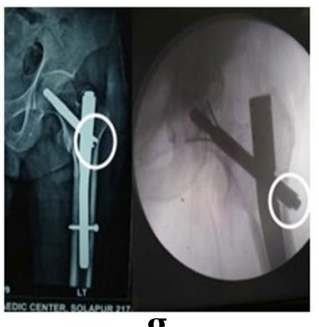

g

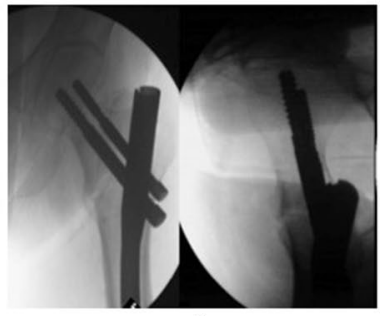

d

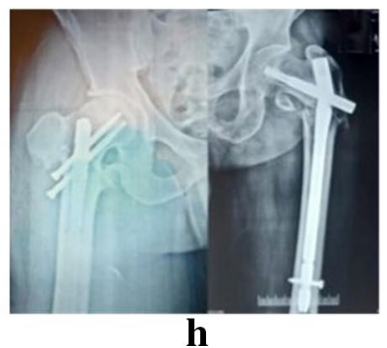

Fig. 1 a Fixing fracture with $2 \mathrm{~K}$ Wires and adducting hip to make nailing easier. b Showing a case done with Positive cortical support, good valgus and good compression leading to a good outcome. $\mathbf{c}$ Various starting entry points. Best nail entry position is Medial to Tip in AP and Centre of the neck in lateral view. d Ideal screw position which inferior to central and parallel to calcar in AP and slightly posterior to centre in lateral with screw tip sub chondrally placed with TAD less than $25 \mathrm{~mm}$. e The cervical screw placed kissing the calcar

One should avoid the entry site getting lateralised, as the bone is weaker on the lateral aspect. While passing the centering awl or the entry reamer, more bone gets removed on outer aspect and the entry site will get lateralised leading to varus happening with the head and neck as well as screw position not proper leading to early cut out of the improperly placed neck screw in a varus head.

4. The neck screw placement It should be placed parallel to the calcar, in the inferior third of the neck in AP view so that the tip of the screw should also be in the inferior part of the head and not at the centre or superior portion of the head. This will allow easy sliding of the neck screw within the nail and causes less jamming. Similarly in the lateral view, it should be in the centre or slightly posterior to the centre so that it has the purchase in the strongest part of the bone (Fig. 1d). For passing these screws properly over guidewire, one should use the trocar first to make the starting hole in the lateral cortex of the bone. The long Guide wires of $1.8-2.5 \mathrm{~mm}$ are not made for drilling the thick lateral cortex like the drill bits and they may slip over the bone especially due to longer lever arm from the other end where drill machine is attached. leading to more TAD, but Cal TAD is good. f Showing fracture not adequately compressed on fracture leading to neck screws backing out in follow up X-ray. $\mathrm{g}$ Back end of headless screw which has gone inside and picture showing it should be out of lateral cortex to allow sliding out during fracture collapse. $\mathbf{h}$ Shorter nail not having fixation at the entry site causing uncontrolled medialisation and another case of non-union and nail breakage due to the same

5. The Neck screw should be placed as subchondral as possible so that Calcar TAD (CalTAD) is less TAD is normally measured from the fovea centralis, which is in the central portion of the head [8]. Since screws are placed as inferior as possible in AP, the screw tip will be definitely away from the fovea centralis and the TAD will be more, in spite of screw being in a very good and strong bone position and in spite of screw tip being placed in a subchondral part of the head. So, author advocate you to measure Cal TAD by extrapolating the screw line where it will touch the articular surface, measure the TAD from that point and not from fovea centralis. This is called as Cal TAD or Calcar TAD [9]. So keep Cal TAD in both AP and lateral views as low as possible and definitely below $25 \mathrm{~mm}$ (Fig. 1e).

6. Compression of fracture on table One should compress the fracture on the table after releasing the traction as much as the fracture needs. The fracture should hardly collapse for the further union after fixation. For this one should measure and use proper length neck screws. If on table traction is not released before final tightening of neck screws, in the immediate post-operative picture itself one can see screws backing out (Fig. 1f). This longer screw having no support of a lateral wall 
to its head can rock in and out and loose purchase. Similarly, if we use a shorter screw, the surgeon may try to pass the screw more deeper so that the tip to reach the subchondral area by extra tightening of the screw, that few extra rotations will spoil the hold of neck screws in bone and they will slide back in due course as well as if the screw is shorter, TAD will be also more in these shorter screws, leading to higher chances of failure. The author feels that fracture needs to be compressed as much as possible after releasing traction on table itself so that fracture need not require the sliding mechanism of the neck screws in the nail in all cases. In few cases where there will be extra resorption at the fracture site only the implants sliding neck screw mechanism should be relied.

7. Tips for headless screw Head less neck screws like Helicle blade of PFN A2 or any other newer single screw system, make sure that the back end of the neck screw remains at least $3-5 \mathrm{~mm}$ outside the lateral cortex so that they can slide out easily when the fracture further collapses during the course of healing. By chance, if they penetrate inside the lateral cortex, during the course fracture collapse, the hole in the bone may not match the neck screw to slide out leading to jamming of sliding out mechanism and hence high penetration chances into the hip (Fig. 1g).

8. Nail projection at entry site The nail has to be little projecting above the entry hole so that the purchase of the nail at this site prevents medialisation of the shaft. If it is buried inside the cancellous bone, it can toggle, migrate medially and can lead to instability, non-union and implant failure (Fig. 1h). It can be addressed by additional end cap over the tip of the nail to build up the nail to project out at the entry site.

9. Static/dynamic locking Though there are many literatures showing good out comes with static locking, but few cases where fracture resorption is a bit more than normal, either they require dynamization by about 6 weeks' time or it can be dynamically locked from day one itself. To be on the safer side, dynamic locking should be done in all nails whenever possible.

10. Choice of implant Finally, good implants from a good manufacturer with laser marking to trace back if complication arises and not a poor copy from a fly by night lesser-known company, costing a bit less should be used to avoid complications related to metal quality and strength of the implant.

Finally, after a very robust and stable fixation, the patient should be mobilised as pain tolerated weight bearing with a walker to enhance early fracture union and to minimise complications of prolonged immobilisation.
Once the patient is made to walk, his morale will be boosted and will cooperate for proper physiotherapy.

Supplementary material The online version of this article (https://doi. org/10.1007/s43465-021-00373-x) contains supplementary material, which is available to authorized users.

Author contributions BS the entire article (editorial) is based on his original work, his ideology and his teaching. SK studied the material (original work) sent by First Author and prepared the manuscript for Editorial in IJO.

\section{Compliance with ethical standards}

Conflict of interest The authors declare that they have no conflict of interest.

Ethical standard statement This article does not contain any studies with human or animal subjects performed by any of the authors.

Informed consent For this type of study informed consent is not required.

\section{References}

1. Bhandari, M., Devereaux, P. J., Tornetta, P., 3rd., Swiontkowski, M. F., Berry, D. J., Haidukewych, G., et al. (2005). Operative management of displaced femoral neck fractures in elderly patients. An international survey. The Journal of Bone and Joint Surgery: American Volume, 87(9), 2122-2130.

2. Lenich, A., Vester, H., Nerlich, M., Mayr, E., Stöckle, U., \& Füchtmeier, B. (2010). Clinical comparison of the second and third generation of intramedullary devices for trochanteric fractures of the hip-blade vs screw. Injury, 41, 1292-1296.

3. Sadowski, C., Lübbeke, A., Saudan, M., Riand, N., et al. (2002). Treatment of reverse oblique and transverse intertrochanteric fractures with use of an intramedullary nail or a 95 degrees screwplate: a prospective, randomized study. The Journal of Bone and Joint Surgery: American Volume, 84A, 372-381.

4. Anglen, J. O., Weinstein, J. N., \& American Board of Orthopaedic Surgery Research Committee. (2008). Nail or plate fixation of intertrochanteric hip fractures: changing pattern of practice. A review of the American Board of Orthopaedic Surgery database. The Journal of Bone and Joint Surgery: American Volume., 90, 700-707.

5. Sanders, D., Bryant, D., Tieszer, C., Lawendy, A.-R., MacLeod, M., Papp, S., et al. (2017). A multicenter randomized control trial comparing a novel intramedullary device (InterTAN) versus conventional treatment (sliding hip screw) of geriatric hip fractures. Journal of Orthopaedic Trauma, 31, 1-8.

6. De Castro, J., Unsay, I. C., Huat, T., \& Kee, E. K. B. (2020). Early experience with the trochanteric fixation nail-advanced (TFN-A): A descriptive review of thirty-four cases from a single center. The Indian Journal of Orthopaedics., 54(Suppl 2), 246-253.

7. Chang, S.-M., Zhang, Y.-Q., Ma, Z., Li, Q., Dargel, J., \& Eysel, P. (2015). Fracture reduction with positive medial cortical support: A key element instability reconstruction for the unstable pertrochanteric hip fractures. Archives of Orthopaedic and Trauma Surgery., 135(6), 811-818.

8. Baumgaertner, M. R., Curtin, S. L., Lindskog, D. M., \& Keggi, J. M. (1995). The value of the tip apex distance in predicting failure 
of fixation of peritrochanteric fractures of the hip. Journal of Bone and Joint Surgery: American Volume, 77, 1058-1064.

9. Aicale, R., \& Maffulli, N. (2018). Greater rate of cephalic screw mobilisation following proximal femoral nailing in hip fractures with a tip-apex distance (TAD) and a calcar referenced TAD greater than $25 \mathrm{~mm}$. Journal of Orthopaedic Surgery and Research., 13, 106.
Publisher's Note Springer Nature remains neutral with regard to jurisdictional claims in published maps and institutional affiliations. 